\title{
APPLICATIONS OF THE $\boldsymbol{u}$-CLOSURE OPERATOR
}

\author{
M. SOLVEIG ESPELIE, JAMES E. JOSEPH AND MYUNG H. KWACK
}

To Professor George H. Butcher on his 60th birthday

\begin{abstract}
Let $\operatorname{cl}_{u}(A)$ be the $u$-closure of a subset $A$ of a space. We prove that a space is compact if and only if for each upper-semicontinuous multifunction $\lambda$ on the space, the multifunction $\mu$ defined on the space by $\mu(x)=\mathrm{cl}_{u}(\lambda(x))$ assumes a maximal value under set inclusion. We also prove that in a Urysohn-closed space any two subsets with disjoint $u$-closures are separated by disjoint open subsets. The quotient space induced by identifying those points with identical $u$-closures is investigated and shown to be $T_{0}$.
\end{abstract}

Introduction. In $\left[\mathbf{H}_{1}\right]$ the notion of $u$-convergence of a filterbase was introduced and utilized to study Urysohn-closed and minimal Urysohn topological spaces in terms of arbitrary filterbases. The notion of $u$-convergence leads naturally to the concept of $u$-closure of a subset $A\left(\mathrm{cl}_{u}(A)\right)$ of a space which was employed in $\left[\mathrm{J}_{1}\right]$ to obtain characterizations of Urysohn-closed and minimal Urysohn spaces. In this paper we (1) define certain subsets of a space in terms of $\mathrm{cl}_{u}$, relate these subsets to others which have recently been studied and establish some decomposition and separation properties for these subsets, (2) show that a space $X$ is compact if and only if for each upper-semicontinuous multifunction $\lambda$ on $X$, the multifunction $\mu$ defined on $X$ by $\mu(x)=\operatorname{cl}_{u}(\lambda(x))$ assumes a maximal value under set inclusion, (3) use the result in (2) to prove a result for the compact-open topology and (4) study the quotient topology induced by identifying those points with identical $u$-closures, using the above results to show that this quotient space is always $T_{0}$ and to gather other information about this space.

Results. Let $X$ be a space, $A \subset X, x \in X$ and let $\Omega$ be a filterbase on $X$. We let $\operatorname{cl}(A)$ and ad $\Omega$ represent the closure of $A$ and adherence of $\Omega$ respectively. We denote by $\Sigma(A)$ the collection of open neighborhoods of $A$ and by $\Lambda(A)$ the collection of open sets which contain closed neighborhoods of $A$. (If $A=\{x\}$, we write $\Sigma(x)$ or $\Lambda(x)$.) The $u$-closure of $A$ is $\{v \in X$ : each $V \in \Lambda(v)$ satisfies $A \cap \operatorname{cl}(V) \neq \varnothing\}$, and the $u$-adherence of $\Omega\left(\operatorname{ad}_{u} \Omega\right)$ is $\cap_{\Omega} \operatorname{cl}_{u}(F)$. The statements in our first proposition will be useful in the sequel. The proof of the proposition is straightforward and is omitted.

Received by the editors February 13, 1980.

AMS (MOS) subject classifications (1970). Primary 54A05, 54D30; Secondary 54B15, 54C60.

Key words and phrases. Compactness, multifunctions, $u$-closure.

(C) 1981 American Mathematical Society 0002-9939/81/0000-0438/\$03.00 
Proposition 1. Let $X$ be a space, let $A \subset X$, let $\Omega$ be a filterbase on $X$ and let $x, y \in X$.

(a) The equations

$$
\operatorname{cl}_{u}(A)=\bigcap_{\Sigma(A)} \operatorname{cl}_{u}(V)=\bigcap_{\Lambda(A)} \operatorname{cl}(W)
$$

and

$$
\operatorname{ad}_{u} \Omega=\operatorname{ad}_{u} \bigcup_{\Omega} \Sigma(F)=\operatorname{ad} \bigcup_{\Omega} \Lambda(F)
$$

hold.

(b) If $x \in \operatorname{cl}_{u}(y)$ then $y \in \operatorname{cl}_{u}(x)$.

A net $g u$-converges to $x$ in a space if $g$ is eventually in $\operatorname{cl}(W)$ for each $W \in \Lambda(x)$ $\left[\mathbf{H}_{1}\right]$. The question is raised in $\left[\mathbf{H}_{1}\right]$ as to how an open filterbase $\Omega$ can be constructed from a net $g$ with the property that some subnet of $g u$-converges to $x$ if and only if $x \in$ ad $\Omega$. In our first theorem this question is answered by appeal to Proposition 1(a) and standard methods.

TheOrem 1. Let $X$ be a space and let $(g, D)$ be 'a net in $X$. For each $n \in D$, let $S(n)=\{g(k): k \geqslant n\}$. Then $\Omega(g)=\cup_{D} \Lambda(S(n))$ is an open filterbase on $X$, and $x \in \operatorname{ad} \Omega(g)$ if and only if some subnet of $g u$-converges to $x$.

We recall [V] that the $\theta$-closure of a subset $A\left(\operatorname{cl}_{\theta}(A)\right)$ of a space is $\{x \in X$ : Each $V \in \Sigma(x)$ satisfies $A \cap \operatorname{cl}(V) \neq \varnothing\}$ and that $\cap_{\Omega} \operatorname{cl}_{\theta}(F)$ is the $\theta$-adherence of a filterbase $\Omega$ on a space. It is readily seen that $\operatorname{cl}_{u}(V)=\operatorname{cl}_{\theta}(\operatorname{cl}(V))$ for any open subset $V$ of a space. Let $X$ be a space and let $A \subset X$. $A$ is quasi $H$-closed $(Q H C)$ relative to $X$ if each filterbase $\Omega$ on $A$ satisfies $A \cap \operatorname{ad}_{\theta} \Omega \neq \varnothing\left[\mathbf{H}_{2}\right]$. We say simply that $A$ is quasi $H$-closed $(Q H C)$ if $A$ is QHC relative to $A$; we define $A$ to be quasi Urysohn-closed (QUC) relative to $X$ if each filterbase $\Omega$ on $A$ satisfies $A \cap \operatorname{ad}_{u} \Omega \neq$ $\varnothing$. It is known that $A$ is $U(i)$ in the sense of Scarborough if and only if $A$ is QUC relative to $A$. It is clear from the definitions and the relationship between $\mathrm{cl}_{u}$ and $\mathrm{cl}_{\theta}$ on open subsets that $A$ is QUC relative to $X$ if $A$ is QHC relative to $X$. Propositions 2 and 3 are stated without proof since the proofs are similar to those of the analogous results for QHC relative subsets.

Proposition 2. The following statements are equivalent for a space $X$ and $A \subset X$.

(a) $A$ is $Q U C$ relative to $X$.

(b) Each open filterbase $\Omega$ on $A$ satisfies $A \cap \operatorname{ad}_{u} \Omega \neq \varnothing$.

(c) $A \cap$ ad $\cup_{\Omega} \Lambda(F) \neq \varnothing$ is satisfied for each filterbase $\Omega$ on $A$.

(d) For each Urysohn open cover $\left[\mathrm{H}_{1}\right], \Delta$, of $A$ by open subsets of $X$, some finite $\Delta^{*} \subset \Delta$ satisfies $A \subset \cup_{\Delta^{*}} \operatorname{cl}(V)$.

(e) Each net $g$ in $A$ has a subnet which u-converges to some point in $A$.

(f) Each filterbase $\Omega$ on $X$ satisfying $F \cap C \neq \varnothing$ for each regular-closed subset which contains $A$ also satisfies $A \cap \operatorname{ad}_{u} \Omega \neq \varnothing$.

(g) Each base for an ultrafilter on $A$ u-converges $\left[\mathbf{H}_{1}\right]$ to some point in $A$. 
We say that a subset $A$ of a space is $u$-closed if $\mathrm{cl}_{u}(A)=A$ and $\theta$-closed if $\operatorname{cl}_{\theta}(A)=A$.

Proposition 3. Let $X$ be a space and let $A \subset X$. If $A$ is $Q U C$ relative to $X$ then $\operatorname{cl}(A)$ is $Q U C$ relative to $X$. If $X$ is $U(i)$ and $A$ is $u$-closed then $A$ is $Q U C$ relative to $\boldsymbol{X}$.

It is known $\left[\mathbf{J}_{2}\right]$ that if $X$ is $\mathrm{QHC}$ then $\operatorname{cl}_{\theta}(A)$ is QHC relative to $X$ for each $A \subset X$. Our next theorem improves this result.

TheOREM 2. If $X$ is $Q H C$ and $A \subset X$ then $\operatorname{cl}_{u}(A)$ is $Q H C$ relative to $X$.

Proof. Let $\Omega$ be a filterbase on $\operatorname{cl}_{u}(A)$. If $W \in \Lambda(A)$, then $\operatorname{cl}_{u}(A) \subset \operatorname{cl}(W)$ and, consequently, $V \cap W \neq \varnothing$ is satisfied for all $V \in \Sigma(F), F \in \Omega$ and $W \in \Lambda(A)$. Hence $\Omega^{*}=\left\{V \cap W: V \in \cup_{\Omega} \Sigma(F), W \in \Lambda(A)\right\}$ is an open filterbase on $X$. Hence $\varnothing \neq \operatorname{ad} \Omega^{*} \subset \operatorname{ad}_{\theta} \Omega \cap \operatorname{cl}_{u}(A)$. The proof is complete.

We leave the following problem open.

Problem. Prove or disprove that $\operatorname{cl}_{u}(A)$ is QUC relative to $X$ for each subset $A$ of a $U(i)$ space.

In the following theorem we present an interesting set inclusion relation between the $\theta$-closure of a QHC relative subset and the $u$-closures of the points of the set.

THEOREM 3. If $A$ is $Q H C$ relative to a space $X$ then $\operatorname{cl}_{\theta}(A) \subset \cup_{A} \operatorname{cl}_{u}(x)$.

Proof. Let $y \in \operatorname{cl}_{\theta}(A)$. Then $A \cap \operatorname{cl}(V) \neq \varnothing$ is satisfied for all $V \in \Sigma(y)$. Hence $\varnothing \neq A \cap \cap_{\Sigma(y)} \operatorname{cl}_{\theta}(\mathrm{cl}(\mathrm{V}))=A \cap \operatorname{ad}_{u} \Sigma(y)=A \cap \mathrm{cl}_{u}(y)$. By Proposition 1(b), $y$ $\in \cup_{A} \mathrm{cl}_{u}(x)$. The proof is complete.

A subset $A$ of a space $X$ is $\theta$-rigid if each filterbase $\Omega$ on $X$ satisfying $F \cap \operatorname{cl}(V) \neq \varnothing$ for all $F \in \Omega$ and $V \in \Sigma(A)$ also satisfies $A \cap \operatorname{ad}_{\theta} \Omega \neq \varnothing[\mathbf{E}-\mathbf{J}]$; we define $A$ to be $u$-rigid if each filterbase $\Omega$ on $X$ satisfying $F \cap \operatorname{cl}(W) \neq \varnothing$ for each $W \in \Lambda(A)$ and $F \in \Omega$ also satisfies $A \cap \operatorname{ad}_{u} \Omega \neq \varnothing$. The following characterization result is stated without proof; $\operatorname{int}(A)$ represents the interior of a subset $A$ of a space.

Proposition 4. The following statements are equivalent for a space $X$ and $A \subset X$.

(a) $A$ is $u$-rigid.

(b) For each Urysohn open cover $\Delta$ of $A$ by open subsets of $X$ some finite $\Delta^{*} \subset \Delta$ satisfies $A \subset \operatorname{int}\left(\cup_{\Delta^{*}} \operatorname{cl}(V)\right)$.

(c) Each open filterbase $\Omega$ on $X$ satisfying $V \cap W \neq \varnothing$ for all $V \in \Omega$ and $W \in \Lambda(A)$ also satisfies $A \cap \operatorname{ad}_{u} \Omega \neq \varnothing$.

(d) If a net in $X$ is frequently in $\mathrm{cl}(W)$ for each $W \in \Lambda(A)$ then some subnet of the net $u$-converges to some point in $A$.

(e) $A \cap$ ad $\cup_{\Omega} \Lambda(F) \neq \varnothing$ is satisfied by any filterbase $\Omega$ on $X$ such that $V \cap W$ $\neq \varnothing$ is satisfied for all $V \in \cup_{\Omega} \Sigma(F)$ and $W \in \Lambda(A)$.

(f) Each base $\beta$ for an ultrafilter on $X$ satisfying $B \cap \operatorname{cl}(W) \neq \varnothing$ for all $B \in \beta$ and $W \in \Lambda(A) u$-converges to a point in $X$. 
Since there are Urysohn-closed spaces which are not $H$-closed, it is clear that there are $u$-rigid subsets which are not $\theta$-rigid. The next result shows that $\theta$-rigid subsets are $u$-rigid.

THEOREM 4. A $\theta$-rigid subset of a space is u-rigid.

Proof. The proof follows from Proposition $4(\mathrm{~b})$ and the recollection that a subset $A$ of a space $X$ is $\theta$-rigid if and only if for each cover $\Delta$ of $A$ by open subsets of $X$ some finite $\Delta^{*} \subset \Delta$ satisfies $A \subset \operatorname{int}\left(\cup_{\Delta^{*}} \operatorname{cl}(V)\right)$.

In our next theorem we give a decomposition result for $u$-rigid subsets.

THEOREM 5. If $A$ is a $u$-rigid subset of a space then $\operatorname{cl}_{u}(A)=\cup_{A} \operatorname{cl}_{u}(x)$.

Proof. Let $y \in \operatorname{cl}_{u}(A)$. Then $y \in \operatorname{cl}(W)$ for each $W \in \Lambda(A)$. Hence $A \cap \operatorname{cl}_{u}(y)$ $\neq \varnothing$ and, consequently, $y \in \cup_{A} \operatorname{cl}_{u}(x)$. The proof of the reverse inclusion is obvious.

Corollary 1. If $A$ is a $\theta$-rigid subset of a space then $\mathrm{cl}_{u}(A)=\cup_{A} \operatorname{cl}_{u}(x)$.

Our next three results provide information on separation of subsets of spaces by disjoint open sets.

THeOReM 6. Two subsets of a $U(i)$ space with disjoint $u$-closures are separated by disjoint open subsets.

Proof. Let $X$ be $U(i)$ and let $A, B$ be subsets of $X$ satisfying $\operatorname{cl}_{u}(A) \cap \operatorname{cl}_{u}(B)=$ $\varnothing$. If all $V \in \Sigma(A)$ and $W \in \Sigma(B)$ satisfy $V \cap W \neq \varnothing$ then $\Omega=\{V \cap W$ : $V \in \Sigma(A), W \in \Sigma(B)\}$ is an open filterbase on $X$. Since $X$ is $U(i)$ we have

$$
\varnothing \neq \operatorname{ad}_{u} \Omega \subset \operatorname{ad}_{u} \Sigma(A) \cap \operatorname{ad}_{u} \Sigma(B)=\operatorname{cl}_{u}(A) \cap \mathrm{cl}_{u}(B) .
$$

This is a contradiction and the proof is complete.

COROllary 2. Two disjoint u-closed subsets of a $U(i)$ space are separated by disjoint open subsets.

THEOREM 7. If $A, B$ are subsets of a space $X$ with $A$ u-rigid and $A \cap \mathrm{cl}_{u}(B)=\varnothing$ then $A$ and $B$ are separated by disjoint open subsets.

Proof. If $\Omega=\{V \cap W: V \in \Sigma(A), W \in \Sigma(B)\}$ is a filterbase on $X$ then, since $A$ is $u$-rigid, we have $A \cap \operatorname{ad}_{u}(\Sigma(B)) \neq \varnothing$. The proof is complete.

Our next two results are preliminary to our characterizations of compactness in terms of $\mathrm{cl}_{u}$ and upper-semicontinuous multifunctions. A multifunction from a set $X$ to a set $Y$ is a function from $X$ to $P(Y)-\{\varnothing\}$, where $P(Y)$ is the family of subsets of $Y$. If $\lambda$ is a multifunction from $X$ to $Y$ we will write $\lambda \in M(X, Y)$ and if $A \subset X$ we write $\lambda(A)$ for $\cup_{A} \lambda(x)$; if $X$ and $Y$ are spaces and $x \in X$, we say that $\lambda$ is upper-semicontinuous (u.s.c.) at $x$ if for each $W \in \Sigma(\lambda(x))$ some $V \in \Sigma(x)$ satisfies $\lambda(V) \subset W ; \lambda$ is upper-semicontinuous (u.s.c.) if $\lambda$ is u.s.c. at each $x \in X$. A multifunction $\lambda \in M(X, Y)$ has a $u$-strongly-closed graph if $\operatorname{ad}_{u} \lambda(\Sigma(x))=\lambda(x)$ for each $x \in X\left[J_{1}\right]$. Each function with a $u$-strongly-closed graph has a stronglyclosed graph [H-L]. 
THEOREM 8. If $\lambda \in M(X, Y)$ is u.s.c. then $\operatorname{ad}_{u} \lambda(\Sigma(x))=\operatorname{cl}_{u}(\lambda(x))$.

Proof. It is clear that $\operatorname{cl}_{u}(\lambda(x)) \subset \operatorname{ad}_{u} \lambda(\Sigma(x))$ and for each $W \in \Sigma(\lambda(x))$, some $V \in \Sigma(x)$ satisfies $\lambda(V) \subset W$ and thus $\operatorname{ad}_{u} \lambda(\Sigma(x)) \subset \operatorname{cl}_{u}(\lambda(x))$. This completes the proof.

COROLlary 3. A u.s.c. multifunction $\lambda$ has a u-strongly-closed graph if and only if $\lambda$ has $u$-closed point images.

THEOREM 9. The following statements are equivalent for a space $X$.

(a) $X$ is compact.

(b) For each u.s.c. multifunction $\lambda$ on $X$ the multifunction $\mu$ on $X$ defined by $\mu(x)=\mathrm{cl}_{u}(\lambda(x))$ assumes a maximal value under set inclusion.

(c) Each u.s.c. multifunction $\lambda$ on $X$ with $u$-closed point images assumes a maximal value under set inclusion.

(d) Each u.s.c. multifunction $\lambda$ on $X$ with a u-strongly-closed graph assumes a maximal value under set inclusion.

Proof. The equivalence of (c) and (d) follows from Corollary 3, and (c) is obviously implied by (b). To establish that (a) implies (b) let $\Omega=\{\mu(x): x \in X\}$ be ordered by set inclusion and let $\Omega^{*}$ be a nonempty chain in $\Omega$. For each $y$ such that $\mu(y) \in \Omega^{*}$ let $F(y)=\{x \in X: \mu(y) \subset \mu(x)\}$. Then $\{F(y)\}$ is a filterbase on the compact space $X$. If $\mu(y) \in \Omega^{*}$ let $v \in \operatorname{cl}(F(y))$ and let $W \in \Sigma(\lambda(v))$. Some $V \in \Sigma(v)$ satisfies $\lambda(V) \subset W$. If $q \in V \cap F(y)$ then $\mu(y) \subset \mu(q)=\operatorname{cl}_{u}(\lambda(q)) \subset$ $\operatorname{cl}_{u}(W)$. Hence $\mu(y) \subset \mu(v), v \in F(y)$ and $F(y)$ is closed. Let $q \in \cap F(y)$. Then $\mu(q)$ is an upper bound for $\Omega^{*}$. By Zorn's Lemma, $\Omega$ has a maximal element. To complete the proof, we will verify that (a) is implied by (c). If $X$ is not compact there is a net $g$ in $X$ with an ordinal $D$ as its index set and no convergent subnet. Let $D$ have the order topology and for each $k \in D$ let $V(k)=X-$ $\operatorname{cl}(\{g(j): j \geqslant k\})$. Then $\{V(k): k \in D\}$ is an increasing open cover of $X$ with no finite subcover. Define $\lambda \in M(X, D)$ by $\lambda(x)=\{j \in D: j<k(x)\}$ where $k(x)$ is the first element $k$ of $D$ with $x \in V(k)$. Since $D$ with the order topology is regular and $\lambda(x)$ is closed for each $x$ then $\mu(x)=\lambda(x)$ for each $x$. If $W \in \Sigma(\lambda(x))$ and $y \in V(k(x))$ then $k(y) \leqslant k(x)$ so that $\lambda(y) \subset \lambda(x) \subset W$. Therefore $\lambda(V(k(x))) \subset$ $W$ and $\lambda$ is u.s.c. Since $\mu$ clearly assumes no maximal value with respect to set inclusion we see that (c) fails. This completes the proof.

In a Urysohn space the $u$-closure of each point is trivially compact and maximal in the set of $u$-closures of points ordered by inclusion. We may use the results in Theorem 9 to prove that in any space the $u$-closures of points satisfy a maximality condition when the $u$-closure of some point is compact.

THEOREM 10. Let $Y$ be a space and let $y_{0} \in Y$ with $\mathrm{cl}_{u}\left(y_{0}\right)$ compact. Then there is a $y \in Y$ such that (a) $\operatorname{cl}_{u}\left(y_{0}\right) \subset \mathrm{cl}_{u}(y)$ and (b) $\mathrm{cl}_{u}(y)$ is maximal in the set of $u$-closures of points when this set is ordered by inclusion.

Proof. Let $X=\left\{y \in Y: \operatorname{cl}_{u}\left(y_{0}\right) \subset \operatorname{cl}_{u}(y)\right\}$. For each $y \in X$ we have $y \in \operatorname{cl}_{u}\left(y_{0}\right)$ from Proposition 1(b). Moreover, if $v \in \operatorname{cl}(X)$ and $W \in \Sigma(v)$ then some $y \in W$ 
satisfies $\operatorname{cl}_{u}\left(y_{0}\right) \subset \operatorname{cl}_{u}(y) \subset \operatorname{cl}_{u}(W)$. Hence $\operatorname{cl}_{u}\left(y_{0}\right) \subset \operatorname{cl}_{u}(v)$ and $X$ is closed in $Y$. Therefore $X$ is a compact subset of $Y$ and since the identity function from $X$ to $Y$ is u.s.c. the proof may be completed by appeal to the fact that (a) implies (b) in Theorem 9.

The proofs of the following easily established corollaries are omitted.

COROLlary 4. If $Y$ is compact then for each $y_{0} \in Y$ there is a $y \in Y$ such that (a) $\operatorname{cl}_{u}\left(y_{0}\right) \subset \operatorname{cl}_{u}(y)$ and $(\mathrm{b}) \mathrm{cl}_{u}(y)$ is maximal in the set of $u$-closures of points when this set is ordered by inclusion.

COROLlaRY 5. If $Y$ is a regular space and $y_{0} \in Y$ then there is a $y \in Y$ such that (a) $y_{0} \in \operatorname{cl}(y)$ and (b) $\operatorname{cl}(y)$ is maximal in the set of closures of points when this set is ordered by inclusion.

If $X$ is a space and $F$ is a collection of functions from $X$ to a space $Y$ with $F$ having the compact-open topology it is known that for each nonempty compact $A \subset X$ the multifunction $H_{A} \in M(F, Y)$ defined by $H_{A}(g)=g(A)$ is u.s.c. so we may prove the following corollary to Theorem 9.

COROLlARY 6. If $X$ and $Y$ are spaces and $F$ is a compact family of functions from $X$ to $Y$ with the compact-open topology then for each nonempty compact $A \subset X$ there is a function $g$ from $X$ to $Y$ such that $\mathrm{cl}_{u}(g(A))$ is maximal with respect to set inclusion.

The quotient space induced by identifying those points of a given space with identical closures has been extensively studied. In the last results in this paper we initiate the study of the quotient space induced by identifying those points of a given space with identical $u$-closures (i.e. we say that $x$ is equivalent to $y$ if $\left.\operatorname{cl}_{u}(x)=\operatorname{cl}_{u}(y)\right)$. For $A \subset X$, let $u[A](u[x]$ if $A=\{x\})$ represent the saturation of $A$ by the equivalence relation (i.e. $u[A]=\{y \in X: y$ is equivalent to some $x \in A\}) . A$ is saturated with the relation if $u[A]=A$. The next proposition follows from previous results.

Proposition 5. The following properties hold for a topological space $X$.

(a) Each $x \in X$ satisfies $u\left[\operatorname{cl}_{u}(x)\right]=\mathrm{cl}_{u}(x)$.

(b) Each u-rigid $A \subset X$ satisfies $u\left[\mathrm{cl}_{u}(A)\right]=\operatorname{cl}_{u}(A)$.

(c) Each $\theta$-rigid $A \subset X$ satisfies $u\left[\mathrm{cl}_{u}(A)\right]=\mathrm{cl}_{u}(A)$.

(d) Each $A \subset X$ satisfies $u[A] \subset \operatorname{cl}_{u}(A)$.

(e) If $A \in \Lambda(B)$ in $X$ then $u[B] \subset \operatorname{cl}(A)$.

(f) For each $x \in X, \cap_{\mathrm{cl}_{u}(x)} \mathrm{cl}_{u}(v)=\left\{y \in X: \mathrm{cl}_{u}(x) \subset \mathrm{cl}_{u}(y)\right\}$.

(g) For $x, y \in X$ the relations (i) $y \in \operatorname{cl}_{u}(x)$, (ii) $u[y] \cap \operatorname{cl}_{u}(x) \neq \varnothing$, (iii) $u[x] \cap$ $\operatorname{cl}_{u}(y) \neq \varnothing$, (iv) $u[x] \subset \operatorname{cl}_{u}(y)$ and (v) $u[y] \subset \operatorname{cl}_{u}(x)$ are equivalent.

Proof. For the proof of (a) let $y \in u\left[\mathrm{cl}_{u}(x)\right]$. There is a $v \in \operatorname{cl}_{u}(x)$ such that $y \in u[v]$. From Proposition 1(b), $x \in \mathrm{cl}_{u}(v)$ and since $\mathrm{cl}_{u}(v)=\mathrm{cl}_{u}(y)$ we obtain $y \in \operatorname{cl}_{u}(x)$. Hence the proof of (a) is complete since $\mathrm{cl}_{u}(x) \subset u\left[\mathrm{cl}_{u}(x)\right]$ from a general property of equivalence relations. The proof that (b) holds follows directly from (a), Theorem 5 and the fact that $u\left[\cup_{\Omega} F\right]=\cup_{\Omega} u[F]$ for any family $\Omega$ of 
subsets of $X$. It is obvious from Corollary 1 and (b) that (c) holds. To prove (d) we note that

$$
u[A]=\bigcup_{A} u[x] \subset \bigcup_{A} u\left[\operatorname{cl}_{u}(x)\right]=\bigcup_{A} \operatorname{cl}_{u}(x) \subset \operatorname{cl}_{u}(A)
$$

for any $A \subset X$. We see that (e) follows from (d) and the readily established fact that $\mathrm{cl}_{u}(B) \subset \operatorname{cl}(A)$ when $A \in \Lambda(B)$. Similar methods may be utilized to establish (f) and (g). The proofs are omitted.

Let $X(\bmod u)$ represent the quotient space induced on $X$ by the equivalence relation obtained. The following result is interesting.

THEOREM 11. $X(\bmod u)$ is $T_{0}$ for any space $X$.

Proof. Suppose $x, y \in X$ with $u[x] \neq u[y]$. Without loss of generality let $v \in$ $\operatorname{cl}_{u}(x)-\operatorname{cl}_{u}(y)$. Then $y \notin \operatorname{cl}_{u}(v)$ and, consequently, $u[y] \cap \operatorname{cl}_{u}(v)=\varnothing$ from Proposition $5(\mathrm{~g})$. Hence $u[y] \subset X-\mathrm{cl}_{u}(v)$ and $u[x] \subset \mathrm{cl}_{u}(v)$. Since $X-\operatorname{cl}_{u}(v)$ is an open subset of $X$ saturated with the relation we conclude that $X(\bmod u)$ is $T_{0}$ and the proof is complete.

If $\mathrm{cl}_{u}(x)$ is maximal in the set of $u$-closures of points when this set is ordered by inclusion it follows that $u[x]=\left\{y \in X: \mathrm{cl}_{u}(x) \subset \mathrm{cl}_{u}(y)\right\}$ and from Proposition 5(f) we have $u[x]$ closed in $X$. Hence we obtain the following proposition and corollaries.

Proposition 6. If $X$ is a space and $\mathrm{cl}_{u}(x)$ is maximal in the set of $u$-closures of points when this set is ordered by inclusion then $u[x]$ is closed in $X$.

COROllaRY 7. If $X$ is a space and $\mathrm{cl}_{u}(x)$ is maximal for all $x \in X$ then $X(\bmod u)$ is $T_{1}$.

COROllary 8. If $X$ is compact then $X(\bmod u)$ has at least one closed singleton.

Proof. Theorem 10 and Proposition 6.

We complete the paper by giving several examples in connection with the preceding results. Let $\{p(k): k=0,1,2,3, \ldots\}$ be a strictly increasing sequence of primes. Let $N$ be the set of natural numbers and let $W=N \cup\{0\}$. For $(j, k, m) \in N \times W \times N$ let $H(j, k, m)=\left\{\left(j+[p(k)]^{-n}, m\right): n \in N\right\}$; now let $J=(W \times N) \cup \cup_{N \times W} H(j, k, 1) \cup \cup_{N \times N \times N} H(j, k, m) \cup\{(0,0),(1,0)\}$ with the topology generated by the aggregate of basic open sets listed below.

(a) Subsets of $J-((W \times N) \cup\{(0,0),(1,0)\})$.

(b) Subsets of the form $\{(0,0)\} \cup \cup_{j>j_{0}} H(j, 0,1)$.

(c) Subsets of the form $\{(1,0)\} \cup \cup_{m>m_{0}} H(j, k, m)$.

(d) Subsets of the form $\{(0, m)\} \cup \cup_{j>j_{0} ; k>k_{0}} H(j, k, m)$.

(e) All relative open sets from the plane in $\{(k, 1): k \in N\} \cup \cup_{N \times W} H(j, k, 1)$.

(f) For $m>1$ and $i \in N$, all sets of the form $A \cup B$ where $A$ is relatively open in $X$ from the plane about $(i, m)$ and $B$ is of the form $\cup_{j \geqslant j_{0}} H(j, i, m-1)$.

$J$ satisfies the following properties.

(a) $J$ is $H$-closed.

(b) The set $\operatorname{cl}_{u}^{n}((0,0))$ fails to be $u$-closed for each $n \in W$. 
(c) $J(\bmod u)$ is not $T_{2}$ even though $\mathrm{cl}_{u}(x)$ is maximal under set inclusion for each $x$. If $x \in J-[(W \times(N-\{1\})) \cup\{(0,0)\}]$, then $\operatorname{cl}_{u}(x)=\{x\} ; \operatorname{cl}_{u}((0,0))=$ $\operatorname{cl}_{u}((0,1))=\{(0,0)\} \cup(N \times\{2\}) \cup\{(0,1)\}=\operatorname{cl}_{u}((n, 2))$ for each $n \in N$; for $m$ $>2, \operatorname{cl}_{u}((0, m-1))=\{(0, m-1)\} \cup(N \times\{m\})=\operatorname{cl}_{u}((j, m))$ for each $j \in N$. Hence $\mathrm{cl}_{u}(x)$ is clearly maximal for each $x \in J$. However, if $V \in \Sigma(u[(0,0)])$ and $A \in \Sigma(u[(0,2)])$ we see that $V \cap A \neq \varnothing$. Thus $J(\bmod u)$ is not $T_{2}$.

(d) Let $V$ be a basic open set about $(0,0)$. Then $u[V]=V \cup(N \times\{2\}) \cup$ $\{(0,1)\}$ which is not open in $J$.

Finally, let $n=1,2,3,4$, let $A(1)$ be the set of primes larger than 9 and let $A(n)$ be the closed interval $[2 n, 2 n+1]$ otherwise. For each $n$, let $\Omega(n)$ be the filter of finite complements on $A(n)$. Let $X=\cup A(n) \cup\{0,1\}$ with the topology generated by the following open set base: $\left\{V \subset X: V\right.$ is a usual open subset of $\left.\cup_{n>2} A(n)\right\}$ $\cup\{\{0\} \cup F(1) \cup F(2) \cup F(3): F(n) \in \Omega(n), n=1,2,3\} \cup\{\{1\} \cup F(3) \cup F(4):$ $F(n) \in \Omega(n), n=3,4\} \cup\{\{p\} \cup F: p \in A(1), F \in \Omega(2)\}$. Then $X$ is compact and $T_{1}$, but $u[11]$ is not closed in $X$ since $0 \in \operatorname{cl}(u[11])-u[11]$.

\section{REFERENCES}

[E-J] M. S. Espelie and J. E. Joseph, Some properties of $\theta$-closure, Canad. J. Math. (to appear).

[H1] L. L. Herrington, Characterizations of Urysohn-closed spaces, Proc. Amer. Math. Soc. 55 (1976), 435-439.

$\left[\mathbf{H}_{2}\right]$ 277-283. , Remarks on H(i) spaces and strongly-closed graphs, Proc. Amer. Math. Soc. 58 (1976),

[H-L] L. L. Herrington and P. E. Long, Characterizations of H-closed spaces, Proc. Amer. Math. Soc. 48 (1975), 469-475.

[J1] J. E. Joseph, On Urysohn-closed and minimal Urysohn spaces, Proc. Amer. Math. Soc. 68 (1978), 235-242.

$\left[\mathbf{J}_{2}\right] \_$, Multifunctions and cluster sets, Proc. Amer. Math. Soc. 74 (1979), 329-337.

[V] N. V. Veličko, H-closed topological spaces, Mat. Sb. 70 (112) (1966), 98-112; English transl., Amer. Math. Soc. Transl. (2) 78 (1968), 103-118.

Department of Mathematics, Howard University, Washington, D. C. 20059 